\title{
Stabilization of a Class of Stochastic Systems with Time Delays
}

\author{
Jian Wang and Cuixia Li \\ School of Mathematics and Statistics, Anyang Normal University, Anyang, Henan 455002, China \\ Correspondence should be addressed to Jian Wang; ss2wangxin@sina.com \\ Received 4 May 2014; Accepted 16 June 2014; Published 2 July 2014 \\ Academic Editor: Shi-Liang Wu \\ Copyright (C) $2014 \mathrm{~J}$. Wang and C. Li. This is an open access article distributed under the Creative Commons Attribution License, \\ which permits unrestricted use, distribution, and reproduction in any medium, provided the original work is properly cited. \\ The problem of exponential stability is investigated for a class of stochastic time-delay systems. By using the decomposition \\ technique and Lyapunov stability theory, two improved exponential stability criteria are derived. Finally, a numerical example \\ is given to illustrate the effectiveness and the benefit of the proposed method.
}

\section{Introduction}

In fact, time delay constantly occurs in the real world, which results in instability of systems. Thus, the stability problem for time-delay systems has been studied for many years $[1-8]$. On the other hand, stochastic modelling has come to play an important role in many fields of science or industry. Stability analysis for stochastic systems has become increasingly meaningful. A number of results have appeared in the literature [9-19]. For instance, in [11], the author provided the criteria for the stability of a class of stochastic systems by Lyapunov theory.

It's worth noting that, the grey systems can be established when parameters are evaluated by grey numbers (see [20]). Until now, there have been a few papers tackling the stability of the systems; some important and innovative results are obtained [20-23]. In [23], the authors provided the delaydependent criteria for exponential robust stability in the forms of nonlinear matrix inequalities and linear matrix inequalities.

In this paper, we deal with the exponential stability for the time-delay grey stochastic systems. By using the method of [21-23] and Lyapunov stability theory, two improved criteria of mean-square exponential stability are proposed. At last, a numerical example is given to verify the criteria.

Notations. $R^{n}$ denotes the $n$ dimensional Euclidean space, the superscript " $T$ " denotes matrix transposition, and the notation $X \geq Y$, where $X$ and $Y$ are symmetric matrices, means that $X-Y$ is positive semidefinite. Let $\left(\Omega, F,\left\{F_{t}\right\}_{t \geq 0}, P\right)$ be a complete probability space with a filtration $\left\{F_{t}\right\}_{t>0}$ satisfying the usual conditions (i.e., it is right continuous and $F_{0}$ contains all $P$-null sets). Let $w(t)=\left(w_{1}(t), \ldots, w_{m}(t)\right)^{T}$ be an $m$-dimensional Brownian motion defined on the probability space. Let $\tau>0$ and $C\left([-\tau, 0] ; R^{n}\right)$ be the family of continuous functions $\varphi$ from $[-\tau, 0]$ to $R^{n}$. Let $L_{F_{0}}^{2}\left([-\tau, 0] ; R^{n}\right)$ be the family of all $F_{0}$-measurable $C\left([-\tau, 0] ; R^{n}\right)$-valued random variables $\xi=\{\xi(\theta):-\tau \leq \theta \leq 0\}$ such that $\sup _{-\tau \leq \theta \leq 0} E|\xi(\theta)|^{2}<\infty$.

\section{Problem Formulation}

Consider

$$
\begin{aligned}
& d x(t)=[A x(t)+B x(t-\tau)+D u(t)] d t \\
& +\sigma(x(t), x(t-\tau), t) d W(t), \quad t \geq 0, \\
& x_{0}=\xi, \quad \xi \in L_{F_{0}}^{2}\left([-\tau, 0] ; R^{n}\right),-\tau \leq t \leq 0 .
\end{aligned}
$$

Definition 1. If there is at least a grey matrix among matrices $A, B$, and $D$ of system (1), then (1) is called grey stochastic time-delay system.

Hence

$$
\begin{gathered}
d x(t)=[A(\otimes) x(t)+B(\otimes) x(t-\tau)+D u(t)] d t \\
+\sigma(x(t), x(t-\tau), t) d W(t), \quad t \geq 0, \\
x_{0}=\xi, \quad \xi \in L_{F_{0}}^{2}\left([-\tau, 0] ; R^{n}\right),-\tau \leq t \leq 0,
\end{gathered}
$$


where $A(\otimes)$ and $B(\otimes)$ are grey matrices and $A(\otimes)=\left(\otimes_{i j}^{a}\right)$, $B(\otimes)=\left(\otimes_{i j}^{b}\right)$.

Clearly, if matrices $A(\otimes)$ and $B(\otimes)$ are replaced by the deterministic matrices $A$ and $B$, the grey system (2) becomes system (1). The equations

$$
\begin{aligned}
& {\left[L_{a}, U_{a}\right]=\left\{A(\widehat{\otimes})=\left(a_{i j}\right): \underline{a_{i j}} \leq a_{i j} \leq \overline{a_{i j}}, i, j=1,2, \ldots, n\right\},} \\
& {\left[L_{b}, U_{b}\right]=\left\{B(\widehat{\otimes})=\left(b_{i j}\right): \underline{b_{i j}} \leq b_{i j} \leq \overline{b_{i j}}, i, j=1,2, \ldots, n\right\}}
\end{aligned}
$$

are said to be the continuous matrix-covered sets of $A(\otimes)$ and $B(\otimes)$.

Definition 2 (see [20]). System (2) is said to be robustly exponentially stable in the mean square, if, for all $\xi \epsilon$ $L_{F_{0}}^{2}\left([-\tau, 0] ; R^{n}\right)$ and arbitrary matrices $A(\widehat{\otimes}) \in\left[L_{a}, U_{a}\right]$, $B(\widehat{\otimes}) \in\left[L_{b}, U_{b}\right]$, there exist scalars $r>0$ and $C>0$, such that

$$
E|x(t, \xi)|^{2} \leq C e^{-r t} \sup _{-\tau \leq \theta \leq 0} E|\xi(\theta)|^{2}, \quad t \geq 0 .
$$

In addition, the following assumptions are made on the system (2).

(H1) $H: R^{n} \times R^{n} \times R_{+} \rightarrow R^{n \times n}$.

(H2) Supposing there exist scalars $\alpha \geq 0, \beta \geq 0$, such that, for $(x, y, t) \in H: R^{n} \times R^{n} \times R_{+}$, the inequality holds, Trace $\left[\sigma^{T}(x, y, t) \sigma(x, y, t)\right] \leq \alpha|x|^{2}+\beta|y|^{2}$.

Before giving the main results, we first present Lemmas 3 and 4 , which are important for the proof of main theorems.

Lemma 3 (see [20]). For arbitrary whitened matrix $A(\widehat{\otimes}) \in$ $\left[L_{a}, U_{a}\right]$, it follows that

(i) $A(\widehat{\otimes})=L_{a}+\Delta A$

(ii) $0 \leq \Delta A \leq U_{a}-L_{a}$

(iii) $\|A(\widehat{\otimes})\|=\left\|L_{a}\right\|+\left\|U_{a}-L_{a}\right\|$,

where $L_{a}=\left(a_{i j}\right)_{m \times n}, U_{a}=\left(\overline{a_{i j}}\right)_{m \times n}, \Delta A=\left(\delta_{i j} \widehat{r}_{i j}\right)_{m \times n}, \delta_{i j}=$ $\overline{a_{i j}}-a_{i j} \geq 0$.

Lemma 4 (see [18]). Let $N$ be a real matrix of appropriate dimensions; for any vectors $x, y \in R^{n}$, one has $2 x^{T} N y \leq$ $\varepsilon x^{T} x+\varepsilon^{-1} y^{T} N^{T} N y$.

\section{Proof of the Main Theorem}

In this section, we discuss the exponential stability for system (2); two improved criteria for robust exponential stability in mean square are proposed.

Theorem 5. System (2) is exponentially robustly stable in mean square. If there exist positive scalars $\varepsilon_{1}, \varepsilon_{2}$, and $\varepsilon_{3}$, such that

$$
\lambda_{\max }\left(L_{a}+L_{a}^{T}\right)+k_{1}+k_{2}<0
$$

here

$$
\begin{aligned}
k_{1}=1 & +\varepsilon_{1}+\varepsilon_{1}^{-1}\left\|U_{a}-L_{a}\right\|^{2}+\varepsilon_{2}+\alpha, \\
k_{2}= & \varepsilon_{2}^{-1}\left(1+\varepsilon_{3}\right) \lambda_{\max }\left(L_{b}^{T} L_{b}\right) \\
& +\varepsilon_{2}^{-1}\left(1+\varepsilon_{3}^{-1}\right)\left\|U_{b}-L_{b}\right\|^{2}+\beta .
\end{aligned}
$$

Then, for all $\xi \in L_{F_{0}}^{2}\left([-\tau, 0] ; R^{n}\right)$, the following inequality holds:

$$
E|x(t, \xi)|^{2} \leq\left(1+k_{2} \tau e^{r \tau}\right) e^{-r t} \sup _{-\tau \leq \theta \leq 0} E|\xi(\theta)|^{2}, \quad t \geq 0,
$$

where $r$ is the unique positive solution of the following equation:

$$
r+\lambda_{\max }\left(L_{a}+L_{a}^{T}\right)+k_{1}+k_{2} e^{r \tau}=0 .
$$

Proof. First, define Lyapunov-Krasovskii functional as follows:

$$
\begin{aligned}
V(x(t), t)= & e^{r t} x^{T}(t) x(t) \\
& +\int_{-\tau}^{0} e^{r(t+\theta)} x^{T}(t+\theta) x(t+\theta) d \theta .
\end{aligned}
$$

Then, we have

$$
\begin{aligned}
L V(x(t), t)= & (r+1) e^{r t} x^{T}(t) x(t) \\
& -e^{r(t-\tau)} x^{T}(t-\tau) x(t-\tau) \\
+ & +e^{r t}\left\{2 x^{T}(t) A(\widehat{\otimes}) x(t)\right. \\
+ & 2 x^{T}(t) B(\widehat{\otimes}) x(t-\tau) \\
+ & \operatorname{Trace}\left[\sigma^{T}(x(t), x(t-\tau), t)\right. \\
& \times \sigma(x(t), x(t-\tau), t)]\} .
\end{aligned}
$$

Using Lemmas 3 and 4, we derive

$$
\begin{aligned}
2 x^{T}( & t) A(\widehat{\otimes}) x(t) \\
\leq & \lambda_{\max }\left(L_{a}+L_{a}^{T}\right) x^{T}(t) x(t) \\
& +\varepsilon_{1} x^{T}(t) x(t)+\varepsilon_{1}^{-1}\left\|U_{a}-L_{a}\right\|^{2} x^{T}(t) x(t), \\
2 x^{T}( & (t) B(\widehat{\otimes}) x(t-\tau) \\
\leq & \varepsilon_{2} x^{T}(t) x(t)+\varepsilon_{2}^{-1}\left(1+\varepsilon_{3}\right) \lambda_{\max } \\
& \times\left(L_{b}^{T} L_{b}\right) x^{T}(t-\tau) x(t-\tau) \\
& +\varepsilon_{2}^{-1}\left(1+\varepsilon_{3}^{-1}\right)\left\|U_{b}-L_{b}\right\|^{2} x^{T}(t-\tau) x(t-\tau) .
\end{aligned}
$$

By assumption (H2), we can obtain

$$
\begin{gathered}
\text { Trace }\left[\sigma^{T}(x(t), x(t-\tau), t) \sigma(x(t), x(t-\tau), t)\right] \\
\leq \alpha x^{T}(t) x(t)+\beta x^{T}(t-\tau) x(t-\tau) .
\end{gathered}
$$


Substituting (11)-(12) into (10), we see that

$$
\begin{aligned}
& L V(x(t), t) \\
& \leq\left[1+r+\lambda_{\max }\left(L_{a}+L_{a}^{T}\right)+\varepsilon_{1}\right. \\
& \left.\quad+\varepsilon_{1}^{-1}\left\|U_{a}-L_{a}\right\|^{2}+\varepsilon_{2}+\alpha\right] e^{r t} x^{T}(t) x(t) \\
& \quad+\left[\varepsilon_{2}^{-1}\left(1+\varepsilon_{3}\right) \lambda_{\max }\left(L_{b}^{T} L_{b}\right)\right. \\
& \left.\quad+\varepsilon_{2}^{-1}\left(1+\varepsilon_{3}^{-1}\right)\left\|U_{b}-L_{b}\right\|^{2}+\beta\right] \\
& \quad \times e^{r t} x^{T}(t-\tau) x(t-\tau) .
\end{aligned}
$$

Using Itô's differential formula and integrating both sides, we obtain

$$
\begin{aligned}
E V(x(t), t) \leq & \sup _{-\tau \leq \theta \leq 0} E|\xi(\theta)|^{2} \\
+ & {\left[1+r+\lambda_{\max }\left(L_{a}+L_{a}^{T}\right)\right.} \\
& \left.+\varepsilon_{1}+\varepsilon_{1}^{-1}\left\|U_{a}-L_{a}\right\|^{2}+\varepsilon_{2}+\alpha\right] \\
& \times \int_{0}^{t} e^{r s} E|x(s)|^{2} d s \\
+ & {\left[\varepsilon_{2}^{-1}\left(1+\varepsilon_{3}\right) \lambda_{\max }\left(L_{b}^{T} L_{b}\right)\right.} \\
& \left.+\varepsilon_{2}^{-1}\left(1+\varepsilon_{3}^{-1}\right)\left\|U_{b}-L_{b}\right\|^{2}+\beta\right] \\
& \times \int_{0}^{t} e^{r s} E|x(s-\tau)|^{2} d s .
\end{aligned}
$$

Moreover, we have

$$
\begin{aligned}
\int_{0}^{t} e^{r s} E|x(s-\tau)|^{2} d s \leq & e^{r \tau} \int_{0}^{t} e^{r u} E|x(u)|^{2} d u \\
& +\tau e^{r \tau} \sup _{-\tau \leq \theta \leq 0} E|\xi(\theta)|^{2} .
\end{aligned}
$$

Combining (14) with (15) and noting the definitions of $k_{1}, k_{2}$, we see that

$$
\begin{aligned}
E V( & x(t), t) \\
\leq & \left(1+k_{2} \tau e^{r \tau}\right) \sup _{-\tau \leq \theta \leq 0} E|\xi(\theta)|^{2} \\
& +\left[r+\lambda_{\max }\left(L_{a}+L_{a}^{T}\right)+k_{1}+k_{2} e^{r \tau}\right] \\
& \times \int_{0}^{t} e^{r s} E|x(s)|^{2} d s .
\end{aligned}
$$

Furthermore,

$$
\begin{aligned}
& f(r)=r+\lambda_{\max }\left(L_{a}+L_{a}^{T}\right)+k_{1}+k_{2} e^{r \tau}, \\
& \text { then } f^{\prime}(r)=1+k_{2} \tau e^{r \tau} .
\end{aligned}
$$

Since $f^{\prime}(r)>0, f(0)=\lambda_{\text {max }}\left(L_{a}+L_{a}^{T}\right)+k_{1}+k_{2}$ and $f(+\infty)=$ $+\infty$.
When (5) holds, (8) must have a unique solution $r>0$. Hence, we have

$$
E|x(t, \xi)|^{2} \leq\left(1+k_{2} \tau e^{r \tau}\right) e^{-r t} \sup _{-\tau \leq \theta \leq 0} E|\xi(\theta)|^{2}, \quad t \geq 0 .
$$

This completes the proof of Theorem 5 .

By a similar approach in [22], whitened system of (2) can be written as

$$
\begin{aligned}
d x(t)=[ & \left(A(\widehat{\otimes})+L_{b}\right) x(t)-L_{b}(x(t)-x(t-\tau)) \\
& +\Delta B x(t-\tau)+D u(t)] d t \\
& +\sigma(x(t), x(t-\tau), t) d W(t) .
\end{aligned}
$$

Since

$$
\begin{aligned}
x(t) & -x(t-\tau) \\
= & \int_{t+\theta}^{t}[A(\widehat{\otimes}) x(s)+B(\widehat{\otimes}) x(s-\tau)+D u(s)] d s \\
& +\int_{t+\theta}^{t} \sigma(x(s), x(s-\tau), s) d W(s), \quad t \geq \tau,
\end{aligned}
$$

then, we can introduce

$$
\begin{aligned}
& H(x, t) \\
& = \begin{cases}\int_{t+\theta}^{t}[A(\widehat{\otimes}) x(s)+B(\widehat{\otimes}) x(s-\tau)] d s \\
\quad+\int_{t+\theta}^{t} \sigma(x(s), x(s-\tau), s) d W(s), & t \geq \tau \\
x(t)-x(t+\theta), & 0 \leq t \leq \tau .\end{cases}
\end{aligned}
$$

Combining (19) and (21) together, whitened system of (2) can be rewritten as

$$
\begin{aligned}
& d x(t)=[ {\left[A(\widehat{\otimes})+L_{b}\right) x(t)-L_{b} H(x, t) } \\
&+\Delta B x(t-\tau)+D u(t)] d t \\
&+\sigma(x(t), x(t-\tau), t) d W(t), \quad t \geq 0, \\
& x_{0}=\xi, \quad \xi \in L_{F_{0}}^{2}\left([-\tau, 0] ; R^{n}\right),-\tau \leq t \leq 0 .
\end{aligned}
$$

Lemma 6. For all $\xi \in L_{F_{0}}^{2}\left([-\tau, 0] ; R^{n}\right)$ and $t>0$, the following inequality holds:

$$
\begin{aligned}
& \int_{0}^{t} e^{r s} E|H(x(s), s)|^{2} d s \\
& \leq\left(k_{1} \tau e^{r \tau}+k_{2} \tau e^{2 r \tau}\right) \int_{0}^{t} e^{r s} E|x(s)|^{2} d s \\
& \quad+k_{2} \tau^{2} e^{2 r \tau} \sup _{-\tau \leq \theta \leq 0} E|\xi(\theta)|^{2}+\sup _{-\tau \leq \theta \leq 0} \psi(\theta),
\end{aligned}
$$


where

$$
\begin{aligned}
l_{1} & =4 \tau\left(\left\|L_{a}\right\|+\left\|U_{a}-L_{a}\right\|\right)^{2}+2 \alpha, \\
l_{2} & =4 \tau\left(\left\|L_{b}\right\|+\left\|U_{b}-L_{b}\right\|\right)^{2}+2 \beta, \\
\psi(\theta) & =\int_{0}^{\tau} e^{r s} E|x(s)-x(s-\tau)|^{2} d s .
\end{aligned}
$$

Proof. First, by the definition of $H(x, t)$, we can derive

$$
\begin{aligned}
& E|H(x(s), s)|^{2} \\
& \leq 2 E\left|\int_{t+\theta}^{t}[A(\widehat{\otimes}) x(s)+B(\widehat{\otimes}) x(s-\tau)] d s\right|^{2} \\
& \quad+2 E\left|\int_{t+\theta}^{t} \sigma(x(s), x(s-\tau), s) d W(s)\right|^{2} .
\end{aligned}
$$

Clearly,

$$
\begin{aligned}
& E\left|\int_{t+\theta}^{t}[A(\widehat{\otimes}) x(s)+B(\widehat{\otimes}) x(s-\tau)] d s\right|^{2} \\
& \leq 2 \tau\left(\left\|L_{a}\right\|+\left\|U_{a}-L_{a}\right\|\right)^{2} \int_{t+\theta}^{t} E|x(s)|^{2} d s \\
& \quad+2 \tau\left(\left\|L_{b}\right\|+\left\|U_{b}-L_{b}\right\|\right)^{2} \int_{t+\theta}^{t} E|x(s-\tau)|^{2} d s .
\end{aligned}
$$

By assumption ( $\mathrm{H} 2)$, we see that

$$
\begin{aligned}
& E\left|\int_{t+\theta}^{t} \sigma(x(s), x(s-\tau), s) d W(s)\right|^{2} \\
& \quad \leq \alpha \int_{t+\theta}^{t} E|x(s)|^{2} d s+\beta \int_{t+\theta}^{t} E|x(s-\tau)|^{2} d s .
\end{aligned}
$$

By (25)-(27) and noting the definitions of $k_{1}, k_{2}$, we can obtain

$$
\begin{aligned}
E|H(t, x(t))|^{2} \leq & k_{1} \int_{t+\theta}^{t} E|x(s)|^{2} d s \\
& +k_{2} \int_{t+\theta}^{t} E|x(s-\tau)|^{2} d s, \quad t \geq \tau .
\end{aligned}
$$

By (28) and integrating both sides, we have

$$
\begin{aligned}
& \int_{0}^{t} e^{r s} E|H(x(s), s)|^{2} d s \\
& \leq \sup _{-\tau \leq \theta \leq 0} \psi(\theta)+k_{1} \int_{\tau}^{t} e^{r s} \int_{s+\theta}^{s} E|x(u)|^{2} d u d s \\
& \quad+k_{2} \int_{\tau}^{t} e^{r s} \int_{s+\theta}^{s} E|x(u-\tau)|^{2} d u d s .
\end{aligned}
$$

Moreover, we can obtain

$$
\begin{array}{r}
\int_{\tau}^{t} e^{r s} \int_{s+\theta}^{s} E|x(u-\tau)|^{2} d u d s \\
\leq \tau e^{2 r \tau} \int_{0}^{t} e^{r s} E|x(s)|^{2} d s \\
\quad+\tau^{2} e^{2 r \tau} \sup _{-\tau \leq \theta \leq 0} E|\xi(\theta)|^{2},
\end{array}
$$

$$
\int_{\tau}^{t} e^{r s} \int_{s+\theta}^{s} E|x(u)|^{2} d u d s \leq \tau e^{r \tau} \int_{0}^{t} e^{r s} E|x(s)|^{2} d s .
$$

Substituting (30) into (29) and noting the definitions of $l_{1}, l_{2}$, and $\psi(\theta),(23)$ holds. The proof of Lemma 6 is completed.

By (22) and Lemma 6, another criterion for system (2) will be given.

Theorem 7. System (2) is exponentially robustly stable in mean square. If there exist positive scalars $\varepsilon_{1}, \varepsilon_{2}$, and $\varepsilon_{3}$, such that

$$
\lambda_{\max }\left(L_{a}+L_{a}^{T}+L_{b}+L_{b}^{T}\right)+m_{1}+m_{2}+m_{3}<0,
$$

here

$$
\begin{aligned}
& m_{1}=1+\varepsilon_{1}+\varepsilon_{1}^{-1}\left\|U_{a}-L_{a}\right\|^{2}+\varepsilon_{2}+\varepsilon_{3}+\alpha, \\
& m_{2}=\varepsilon_{3}^{-1}\left\|U_{b}-L_{b}\right\|^{2}+\beta, \\
& m_{3}=\varepsilon_{2}^{-1}\left(l_{1}+l_{2}\right) \tau \lambda_{\max }\left(L_{b}^{T} L_{b}\right) .
\end{aligned}
$$

Then, for all $\xi \in L_{F_{0}}^{2}\left([-\tau, 0] ; R^{n}\right)$, the following inequality holds:

$$
\begin{aligned}
E|x(t, \xi)|^{2} \leq[ & \left(1+m_{2} \tau e^{r \tau}+\varepsilon_{2}^{-1} l_{2} \tau^{2} e^{2 r \tau} \lambda_{\max }\left(L_{b}^{T} L_{b}\right)\right) \\
& \quad \times \sup _{-\tau \leq \theta \leq 0} E|\xi(\theta)|^{2} \\
& \left.+\varepsilon_{2}^{-1} \lambda_{\max }\left(L_{b}^{T} L_{b}\right) \sup _{-\tau \leq \theta \leq 0} \psi(\theta)\right] e^{-r t}
\end{aligned}
$$

where $r$ is the unique positive solution of the following equation:

$$
\begin{aligned}
r+\lambda_{\max } & \left(L_{a}+L_{a}^{T}+L_{b}+L_{b}^{T}\right)+m_{1}+m_{2} e^{r \tau} \\
& +\varepsilon_{2}^{-1}\left(l_{1} \tau e^{r \tau}+l_{2} \tau e^{2 r \tau}\right) \lambda_{\max }\left(L_{b}^{T} L_{b}\right)=0 .
\end{aligned}
$$

Proof. Similar to the proof process of Theorem 5, we can derive

$$
\begin{aligned}
L V(x(t), t) \leq & {\left[r+\lambda_{\max }\left(L_{a}+L_{a}^{T}+L_{b}+L_{b}^{T}\right)+m_{1}\right] } \\
& \times e^{r t} x^{T}(t) x(t) \\
& +m_{2} e^{r t} x^{T}(t-\tau) x(t-\tau) \\
& +\varepsilon_{2}^{-1} \lambda_{\max }\left(L_{b}^{T} L_{b}\right) e^{r t}|H(x(t), t)|^{2}
\end{aligned}
$$


Using Itô's differential formula and integrating and by the definition of $H(x, t)$, we obtain

$$
\begin{aligned}
E V(x(t), t) \leq[ & +\lambda_{\max }\left(L_{a}+L_{a}^{T}+L_{b}+L_{b}^{T}\right)+m_{1} \\
& +m_{2} e^{r t}+\varepsilon_{2}^{-1}\left(l_{1} \tau e^{r \tau}+l_{2} \tau e^{2 r \tau}\right) \\
& \left.\times \lambda_{\max }\left(L_{b}^{T} L_{b}\right)\right] \\
\times & \int_{0}^{t} e^{r s} E|x(s)|^{2} d s \\
+ & {\left[1+m_{2} \tau e^{r \tau}\right.} \\
& \left.+\varepsilon_{2}^{-1} l_{2} \tau^{2} e^{2 r \tau} \lambda_{\max }\left(L_{b}^{T} L_{b}\right)\right] \sup _{-\tau \leq \theta \leq 0} E|\xi(\theta)|^{2} \\
+ & \varepsilon_{2}^{-1} \lambda_{\max }\left(L_{b}^{T} L_{b}\right) \sup _{-\tau \leq \theta \leq 0} \psi(\theta) .
\end{aligned}
$$

If $g(r)=r+\lambda_{\text {max }}\left(L_{a}+L_{a}^{T}+L_{b}+L_{b}^{T}\right)+m_{1}+m_{2} e^{r \tau}+\varepsilon_{2}^{-1}\left(l_{1} \tau e^{r \tau}+\right.$ $\left.l_{2} \tau e^{2 r \tau}\right) \lambda_{\max }\left(L_{b}^{T} L_{b}\right)$, then $g^{\prime}(r)=1+m_{2} \tau e^{r \tau}+\varepsilon_{2}^{-1}\left(l_{1} \tau^{2} e^{r \tau}+\right.$ $\left.2 l_{2} \tau^{2} e^{2 r \tau}\right) \lambda_{\max }\left(L_{b}^{T} L_{b}\right)$.

Because $g^{\prime}(r)>0, g(0)=\lambda_{\max }\left(L_{a}+L_{a}^{T}+L_{b}+L_{b}^{T}\right)+m_{1}+$ $m_{2}+m_{3}$ and $g(+\infty)=+\infty$.

If (31) holds, (34) must have a unique solution $r>0$.

Therefore, we can easily get

$$
\begin{aligned}
E V(x(t), t) \leq & {\left[1+m_{2} \tau e^{r \tau}+\varepsilon_{2}^{-1} l_{2} \tau^{2} e^{2 r \tau} \lambda_{\max }\left(L_{b}^{T} L_{b}\right)\right] } \\
& \times \sup _{-\tau \leq \theta \leq 0} E|\xi(\theta)|^{2} \\
& +\varepsilon_{2}^{-1} \lambda_{\max }\left(L_{b}^{T} L_{b}\right) \sup _{-\tau \leq \theta \leq 0} \psi(\theta),
\end{aligned}
$$

or equivalently (33) holds; the proof of Theorem 7 is completed.

\section{Examples}

Consider a stochastic time-delay system

$$
\begin{gathered}
d x(t)=[A(\otimes) x(t)+B(\otimes) x(t-0.5)] d t \\
+\sigma(x(t), x(t-0.5), t) d W(t), \\
x_{0}=\xi, \quad \xi \in L_{F_{0}}^{2}\left([-0.5,0] ; R^{2}\right),-0.5 \leq t \leq 0,
\end{gathered}
$$

where

$$
\begin{array}{rlr}
L_{a}=\left[\begin{array}{cc}
-3.35 & 0.22 \\
0.23 & -3.34
\end{array}\right] ; & U_{a}=\left[\begin{array}{cc}
-3.15 & 0.32 \\
0.31 & -3.45
\end{array}\right] \\
L_{b}=\left[\begin{array}{cc}
-1.15 & 0.20 \\
0.23 & -1.16
\end{array}\right] ; & U_{b}=\left[\begin{array}{cc}
-1.12 & 0.22 \\
0.31 & -1.09
\end{array}\right] .
\end{array}
$$

Respectively, $L_{a}, U_{a}, L_{b}$, and $U_{b}$ are the lower bound and upper bound matrices of $A(\otimes)$ and $B(\otimes)$.
In addition,

$$
\sigma(x(t), x(t-0.5), t)=\left[\begin{array}{l}
\frac{1}{2} x_{1}(t) \sin \left(x_{2}(t-0.5)\right) \\
\frac{1}{2} x_{2}(t) \sin \left(x_{1}(t-0.5)\right)
\end{array}\right] .
$$

Clearly,

$$
\begin{aligned}
& \text { Trace }\left[\sigma^{T}(x(t), x(t-0.5), t) \sigma(x(t), x(t-0.5), t)\right] \\
& \quad \leq 0.25 x^{2}(t) .
\end{aligned}
$$

By using the method of [21], we can obtain that $r=1.7513$ or $r=1.8162$, which indicates that the system (38) is exponentially stable in mean square.

\section{Conclusion}

In this paper, we have investigated a class of grey stochastic systems with time delay; by constructing a suitable LyapunovKrasovskii functional combined with Itô's differential formula, two improved exponential stability criteria are derived. The criteria obtained in this paper are so conveniently verified that the results in this paper should be proved to be very useful in applications.

\section{Conflict of Interests}

The authors declare that there is no conflict of interests regarding the publication of this paper.

\section{Acknowledgments}

The research is supported by the National Natural Science Foundation of China (no. 11301009) and the Natural Science Foundation of Henan Province (no. 0511013800).

\section{References}

[1] S. Arik, "Global robust stability analysis of neural networks with discrete time delays," Chaos, Solitons and Fractals, vol. 26, no. 5, pp. 1407-1414, 2005.

[2] E. K. Boukas, Z. K. Liu, and G. X. Liu, "Delay-dependent robust stability and $H_{\infty}$ control of jump linear systems with timedelay," International Journal of Control, vol. 74, no. 4, pp. 329340, 2001.

[3] Y. S. Lee, Y. S. Moon, W. H. Kwon, and P. G. Park, "Delaydependent robust $\mathrm{H}_{\infty}$ control for uncertain systems with a state-delay," Automatica, vol. 40, no. 1, pp. 65-72, 2004.

[4] J. H. Park, "Robust stabilization for dynamic systems with multiple time-varying delays and nonlinear uncertainties," Journal of Optimization Theory and Applications, vol. 108, no. 1, pp. 155174, 2001.

[5] P. Park, "A delay-dependent stability criterion for systems with uncertain time-invariant delays," IEEE Transactions on Automatic Control, vol. 44, no. 4, pp. 876-877, 1999.

[6] E. K. Boukas and Z. K. Liu, "Robust stability and stabilizability of Markov jump linear uncertain systems with mode-dependent time delays," Journal of Optimization Theory and Applications, vol. 109, no. 3, pp. 587-600, 2001. 
[7] W. Xiong and J. Liang, "Novel stability criteria for neutral systems with multiple time delays," Chaos, Solitons and Fractals, vol. 32, no. 5, pp. 1735-1741, 2007.

[8] J. Cao and J. Wang, "Global exponential stability and periodicity of recurrent neural networks with time delays," IEEE Transactions on Circuits and Systems I: Regular Papers, vol. 52, no. 5, pp. 920-931, 2005.

[9] A. V.Skorokhod, Asymptotic Methods in the Theory of Stochastic Differential Equations, American Mathematical Society, Providence, RI, USA, 1989.

[10] L. Huang and X. Mao, "Robust delayed-state-feedback stabilization of uncertain stochastic systems," Automatica, vol. 45, no. 5, pp. 1332-1339, 2009.

[11] E. I. Verriest, "Stochastic stability of a class of distributed delay systems," in Proceedings of the 44th IEEE Conference on Decision and Control, and the European Control Conference (CDC-ECC '05), pp. 5048-5053, Seville, Spain, December 2005.

[12] T. Shaikhet, "Stability of stochastic hereditarty systems with Markov switching," Theory of Stochastic Processes, vol. 18, no. 2, pp. 180-184, 1996.

[13] S. Xu, J. Lam, X. Mao, and Y. Zou, "A new LMI condition for delay-dependent robust stability of stochastic time-delay systems," Asian Journal of Control, vol. 7, no. 4, pp. 419-423, 2005.

[14] S. Xu, J. Lam, and D. W. C. Ho, "Novel global robust stability criteria for interval neural networks with multiple time-varying delays," Physics Letters, Section A: General, Atomic and Solid State Physics, vol. 342, no. 4, pp. 322-330, 2005.

[15] H. Yan, X. Huang, H. Zhang, and M. Wang, "Delay-dependent robust stability criteria of uncertain stochastic systems with time-varying delay," Chaos, Solitons and Fractals, vol. 40, no. 4, pp. 1668-1679, 2009.

[16] X. Mao, N. Koroleva, and A. Rodkina, "Robust stability of uncertain stochastic differential delay equations," Systems \& Control Letters, vol. 35, no. 5, pp. 325-336, 1998.

[17] L. Wan and J. Sun, "Mean square exponential stability of stochastic delayed Hopfield neural networks," Physics Letters A: General, Atomic and Solid State Physics, vol. 343, no. 4, pp. 306318, 2005.

[18] A. Friedman, Stochastic Differential Equations and their Applications, Academic Press, New York, NY, USA, 1976.

[19] Z. Wang and H. Qiao, "Robust filtering for bilinear uncertain stochastic discrete-time systems," IEEE Transactions on Signal Processing, vol. 50, no. 3, pp. 560-567, 2002.

[20] C. H. Su and J. J. Li, "Research on stability of grey neutral stochastic linear delay systems," Acta Analysis Functionalis Applicata, vol. 12, no. 4, pp. 328-334, 2010.

[21] C. H. Su and S. F. Liu, "Robust stability of grey stochastic nonlinear systems with distributed delays," Mathematics in Practice and Theory, vol. 38, no. 22, pp. 218-223, 2008.

[22] C. H. Su and S. F. Liu, "Mean-square Exponential Robust Stability for a Class of Grey Stochastic Syst em s with Distr ib uted Delays," Journal of Xinyang Normal University, vol. 23, no. 4, pp. 501-505, 2010.

[23] C. H. Su and S. F. Liu, "Exponential robust stability of grey neutral stochastic systems with distributed delays," Chinese Journal of Engineering Mathematics, vol. 27, no. 3, pp. 403-414, 2010. 


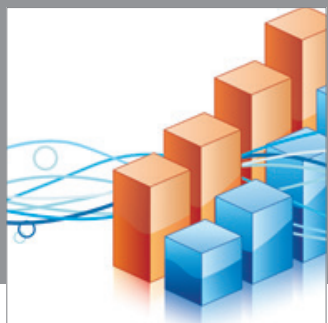

Advances in

Operations Research

mansans

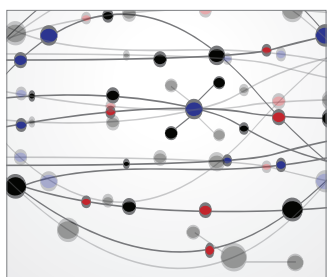

The Scientific World Journal
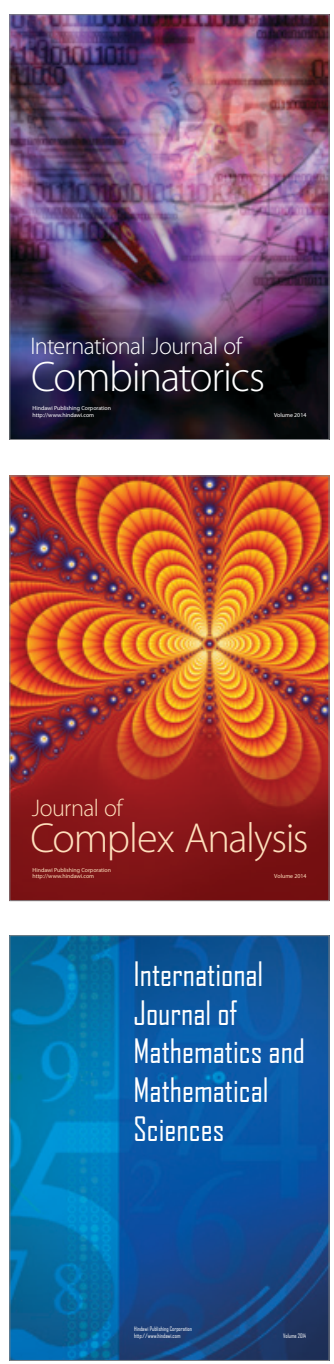
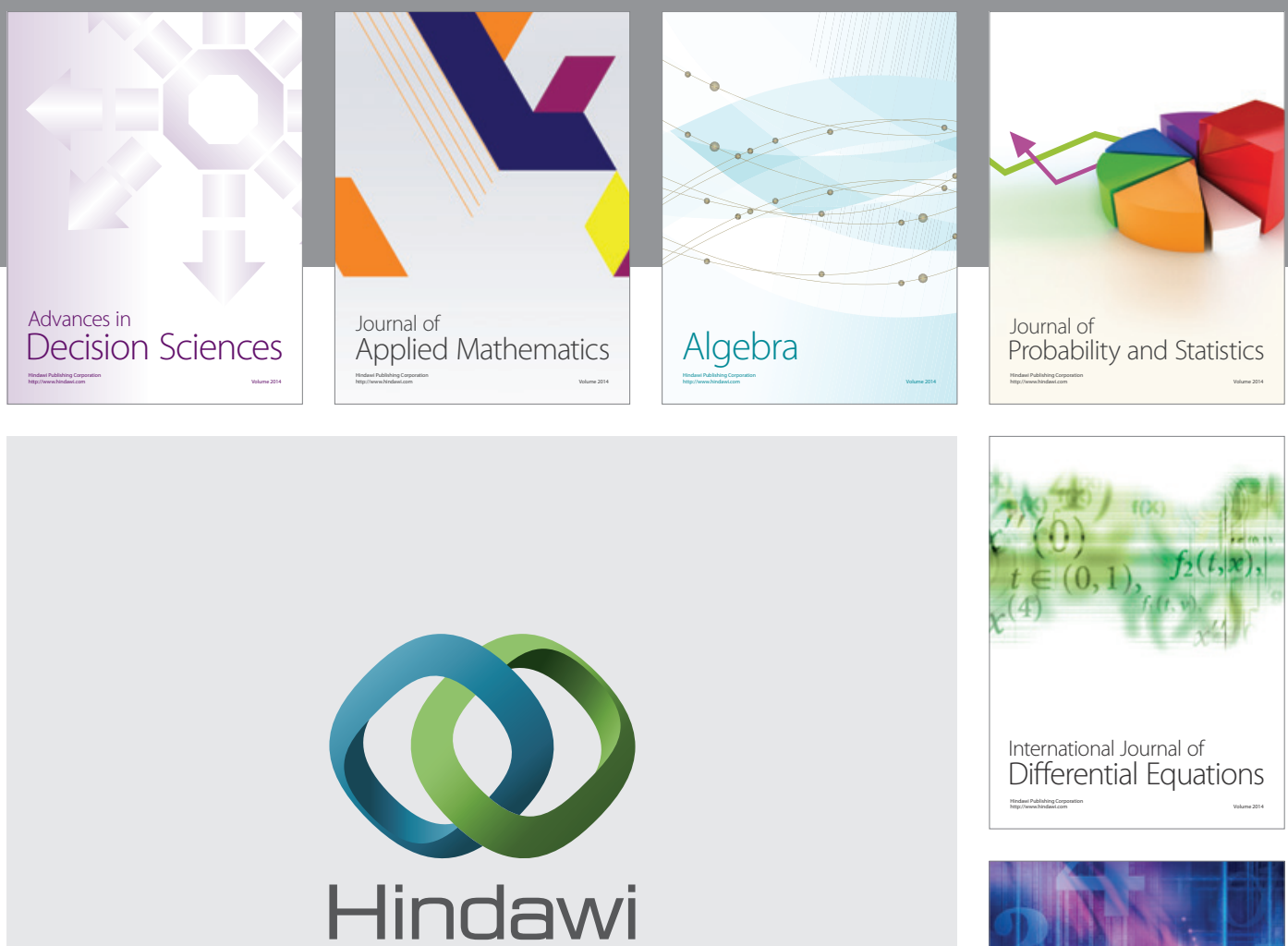

Submit your manuscripts at http://www.hindawi.com
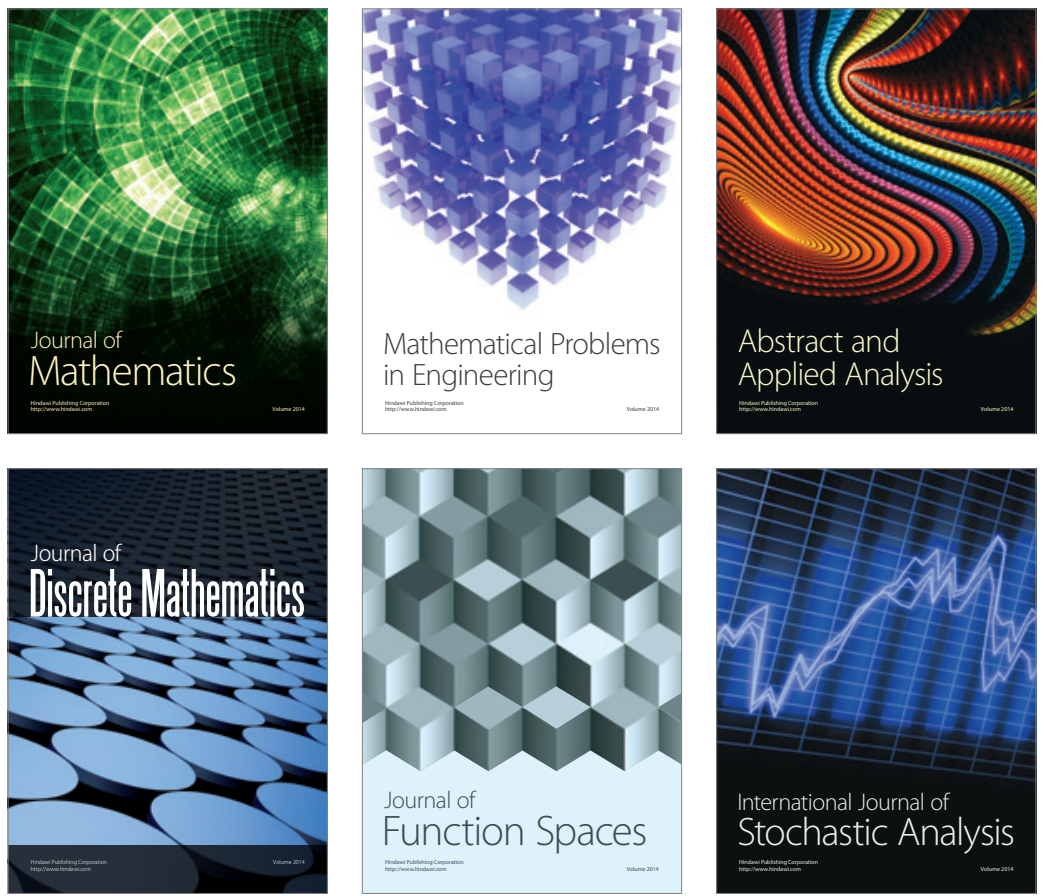

Journal of

Function Spaces

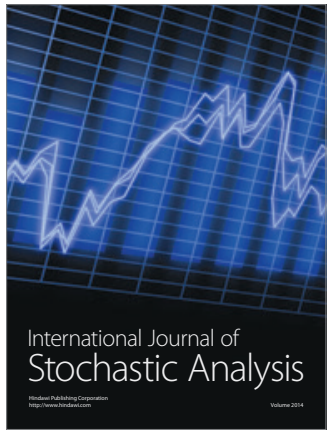

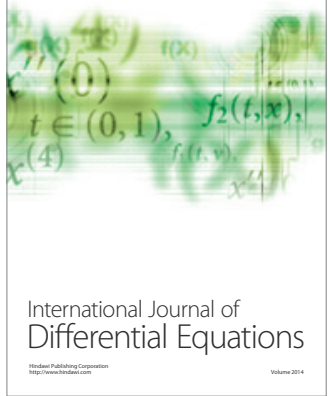
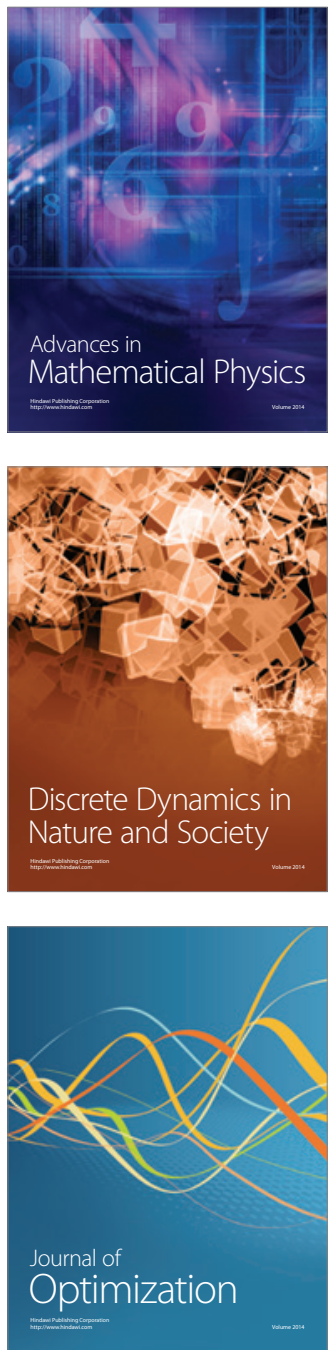